Dom. Cien., ISSN: 2477-8818

Vol. 4, núm.4., oct, 2018, pp. 373-386

Prevalencia y factores de riesgo en enfermedades cardiovasculares: Hipertensión Arterial

\title{
Prevalencia y factores de riesgo en enfermedades cardiovasculares: Hipertensión Arterial
}

\section{Prevalence and risk factors in cardiovascular diseases: Arterial Hypertension}

\section{Prevalência e fatores de risco nas doenças cardiovasculares: Hipertensão arterial}

\author{
Margarita de Jesús Tobar-Solórzano ${ }^{\mathrm{I}}$ \\ margarita-1jesus@gmail.com \\ Luz María del Carmen Rodríguez-Cepeda ${ }^{\text {II }}$ \\ luzroodríguez1@gmail.com \\ Irene Astudillo-García ${ }^{\text {III }}$ \\ irene_astudillo@hotmail.com
}

\author{
Holanda D. Vizcaino-Cevallos IV \\ holanda_vizcai@hotmail.com \\ María D. Ayala Astudillo v \\ maria_ayala12@hotmail.com \\ Víctor F. Carvajal Barahona VI \\ victor.carvajal12@yahoo.com
}

Recibido: 07 de septiembre de 2018 * Corregido: 28 de septiembre de 2018 * Aceptado: 25 de octubre de 2018

I. Especialista en Enfermería de Cuidados Críticos; Licenciada en Enfermería, Universidad Estatal Península de Santa Elena Santa Elena, Ecuador.

II. Licenciada en Enfermería, Especialista en Enfermería en cuidados Críticos; Labora en la Universidad Estatal de Guayaquil, Guayaquil, Ecuador.

III. Licenciada en Enfermería, Master en Gerencia en Salud, Docente del Instituto Superior Tecnológico Bolivariano, Guayaquil, Ecuador

IV. Licenciada en Enfermería; Magister en Gerencia en Salud para el Desarrollo Local en Salud, Docente del Instituto Superior Tecnológico Bolivariano, Guayaquil, Ecuador.

v. Licenciada en Nutrición Dietética y Estética de la Universidad Católica de Guayaquil; Docente y Coordinadora de la carrera de Tricología y Cosmiatria del Instituto Tecnológico Bolivariano de Guayaquil, Guayaquil, Ecuador.

VI. Médico Cirujano; Hospital IESS Portoviejo, Portoviejo, Ecuador. 


\section{Resumen}

La hipertensión arterial es un tema de salud pública, ya que se ha demostrado que en grupos de personas mayores a 45 años de edad, el 90\% de los casos desarrolla la hipertensión esencial o primaria y menos del 10\% desarrolla hipertensión arterial secundaria. Teniendo esto en mente es de suma importancia conocer los factores de riesgo tradicionales modificables y no modificables que influyen en el desarrollo de la enfermedad.

La proporción de probabilidades expresa que el fumar, el índice de masa corporal, la diabetes mellitus y la dislipidemia son factores asociados a la hipertensión arterial. La hipertensión y su asociación con otros factores de riesgo cardiovasculares tales como diabetes, obesidad y dislipidemia muestran la necesidad de intervenciones de políticas públicas y de estado además de la implementación de protocolos enfocados en minimizar las complicaciones que surgen derivadas de este flagelo que no distingue de edades ni clases sociales y se ha convertido en uno de las mayores causas de muertes en los últimos años.

Palabras claves: Enfermedades cardiovasculares, Hipertension arterial, Salud. 


\section{Abstract}

Hypertension is a public health issue, since it has been shown that in groups of people over 45 years of age, $90 \%$ of cases develop essential or primary hypertension and less than $10 \%$ develop secondary hypertension. Bearing this in mind, it is very important to know the modifiable and nonmodifiable traditional risk factors that influence the development of the disease.

The proportion of probabilities expresses that smoking, body mass index, diabetes mellitus and dyslipidemia are factors associated with arterial hypertension. Hypertension and its association with other cardiovascular risk factors such as diabetes, obesity and dyslipidemia show the need for public policy and state interventions in addition to the implementation of protocols focused on minimizing the complications arising from this scourge that does not distinguish ages or social classes and has become one of the biggest causes of deaths in recent years.

Key words: Cardiovascular diseases, Hypertension, Health. 


\section{Introducción.}

La hipertensión arterial (HTA) forma parte del grupo de enfermedades cardiovasculares que simbolizan la mayor proporción de enfermedades causales de mortalidad como el accidente cerebro vascular (ACV) e infarto agudo de miocardio, que alcanza aproximadamente las dos quintas partes de la población adulta en países desarrollados (Ferreira \& Aydos, 2010). De esta manera, existe una necesidad de evaluar los factores de riesgo que contribuyen a esta condición clínica y su prevalencia, la identificación de grupos en mayor riesgo de ser afectado por hipertensión arterial significa una importante contribución a la prevención de las morbilidades y efectividad del tratamiento.

Hoy se sabe que la hipertensión arterial detectada en algunos niños pueden ser efectos secundarios de enfermedades renales, por ejemplo, Pero también en otros casos puede denotar el inicio precoz de la hipertensión arterial observada en adultos.

Entre la etiología determinante para la elevación de los valores en la presión de sangre (PA), está claro que tanto la genética como la los factores ambientales juegan un papel importante. De esta manera, desde que la hipertensión arterial involucra estos factores, se clasifica como un complejo de enfermedad multifactorial (Mariana \& Pereira, 2014). Dada la alta prevalencia de la hipertensión arterial es esencial el monitoreo y control de los agentes causales. La mayoría de los pacientes hipertensos tienen factores de riesgo cardiovascular modificables como la diabetes mellitus (DM), sobrepeso, estilo de vida sedentario, hábitos de fumar, ingesta de sal, entre otros.

\footnotetext{
376 Vol. 4, núm. 4, octubre 2018, pp. 373-386 
Es necesario sensibilizar a la población para adoptar un sano estilo de vida, no solo una dieta equilibrada y la práctica de actividad física regular, pero también por el seguimiento frecuente de la PA.

Las enfermedades cardiovasculares (ECV) que principalmente incluyen enfermedad coronaria (CHD), accidente cerebrovascular, enfermedad cardíaca reumática (RHD) y la miocardiopatía representa la principal causa de muerte en todo el mundo. A principios del siglo XX, las ECV fueron responsable al menos del 10\% de todas las muertes en todo el mundo, pero aumentó a $30 \%$ en 2001 . Se estima que para el 2020 , ECV se convertirá en la principal causa de muerte y discapacidad en países con ingresos medios y bajos (Murray \& Lopez, 1996).

Estudio del corazón de Framingham en 1961 fue el primero en introducir el concepto de factores de riesgo que vinculan la presencia de colesterol alto, consumo de tabaco, hipertensión y diabetes mellitus a futuras enfermedades cardiovasculares.

El cuadro de predicción de riesgo para ECV de la Organización Mundial de la Salud (OMS) y la Sociedad Internacional de Hipertensión (ISH), diseñado en 2007, proporciona un enfoque simplificado para identificar personas con alto riesgo de ECV en 14 subregiones epidemiológicas de la OMS. El gráfico de predicción de riesgo de la OMS / ISH considera seis determinantes principales de la ECV: edad, sexo, estado de fumador, estado de diabetes mellitus, presión arterial sistólica y nivel de colesterol. También se ha informado que su uso es eficiente en los 193 estados miembros de la OMS, particularmente en países que actualmente no tienen su propio gráfico de predicción (OMS, 2007).

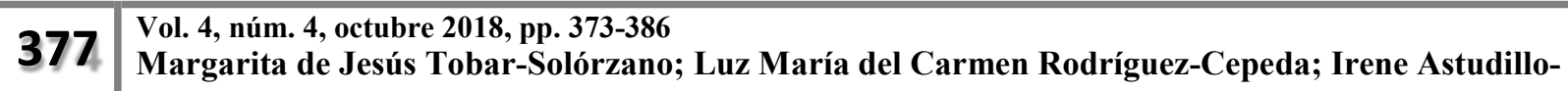
García; Holanda D. Vizcaino-Cevallos; María D. Ayala Astudillo; Víctor F. Carvajal Barahona
} 
Esta revisión tiene como objetivo evaluar la prevalencia de la hipertensión arterial e identificar sus factores de riesgo en pacientes hipertensos de todas las edades.

\section{Metodología.}

La revisión se ha centrado en textos, documentos y artículos científicos publicados disponibles en la web, considerando que aquella herencia de la globalización nos permite acceder a mayor y mejor información a través de las herramientas tecnológicas. Los criterios de inclusión se basaron en publicaciones del habla hispana e inglesa y visible en bases de datos que nos aportaron en la historia y evolución de investigación. El motor de búsqueda ha sido herramientas académicas de la web que direccionan específicamente a archivos con validez y reconocimiento científico, descartando toda información no confirmada o sin las respectivas referencias bibliográficas.

\section{Resultados.}

La Organización Mundial de la Salud define la hipertensión arterial conocida como presión alta o elevada, como una condición en la cual los vasos sanguíneos han aumentado la presión de forma persistente. También afirma que cuanto mayor es la presión, más difícil es contraer el corazón. Sin embargo, muchos autores tienen su propia definición como: un síndrome caracterizado por niveles elevados de presión arterial, asociado a anomalías metabólicas, hormonales y fenómeno trófico (hipertrofia cardíaca y vascular) (Salgado, 2007); Una entidad clínica multifactorial, caracterizada por la presencia de presión arterial sistólica (SAP) igual o persistentemente por encima de $140 \mathrm{mmHg}$ y / o nivel de presión arterial diastólica (PAD), consistentemente igual o superior a 90 mmHg y enfermedad crónica silenciosa y de evolución lenta. La presión sanguinea tiene dos medidas: Presión arterial sistólica o "máxima" y la presión arterial diastolica o "mínimo". El primero

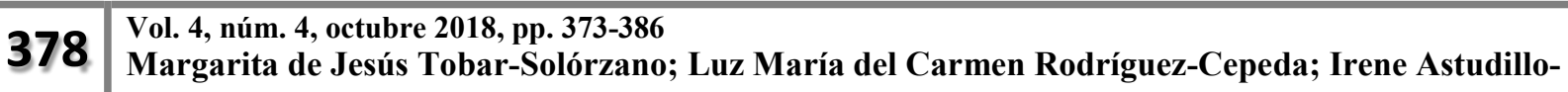
García; Holanda D. Vizcaino-Cevallos; María D. Ayala Astudillo; Víctor F. Carvajal Barahona
} 
corresponde al momento en que el corazón contrae, enviando sangre alrededor del cuerpo y El segundo sucede cuando el corazón se relaja para llenarse de sangre de nuevo.

Para la población adulta, la definición de hipertensión arterial es epidemiológico, por lo que se considera anormal la presión arterial de un adulto cuando está por encima de un nivel en el que hay una asociación con enfermedad coronaria, ACV o enfermedad renal (Moura, Silva, \& Silva, 2015). En contraste, para niños y adolescentes la definición es estadística, esto porque no hay estudios para determinar cuáles serían los niveles de presión arterial asociados con enfermedades futuras.

Para entender la génesis de esta enfermedad, hay que saber que la presión arterial ejercida por la sangre dentro de las arterias varía según la cambios fisiológicos en el sistema cardiovascular, en particular en relación con el gasto cardíaco y la resistencia vascular periférica. [1]. Además, todo este complejo proceso puede sufrir variaciones a lo largo del día oscilando a valores menores o mayores, según el requisito de cada ocasión. Así, es considera que una persona es hipertensa cuando presenta, en al menos en dos ocasiones diferentes, uno de los valores de presión arterial. (sistólica o diastólica) o ambas iguales o superiores a 140/90 $\mathrm{mmHg}$, determinado por un profesional capacitado y utilizando un calibrado y dispositivo validado.

La presión arterial evaluada debe compararse con la referencia de valores adoptados, que definen los límites según género, edad y percentil de altura, utilizando marcos de referencia (Salgado, 2007).

Algunos autores aún consideran la existencia de la "hipertensión arterial de bata blanca", definiéndose como una presión arterial (PA) persistentemente elevada en la consulta y una PA

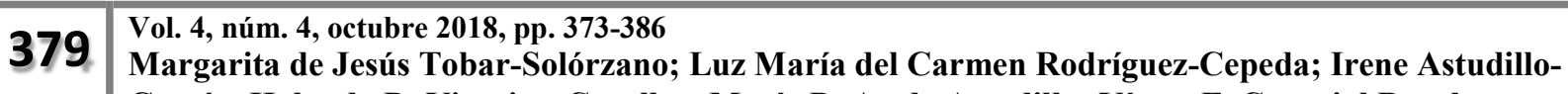
García; Holanda D. Vizcaino-Cevallos; María D. Ayala Astudillo; Víctor F. Carvajal Barahona
} 
ambulatoria de 24 horas inferior a $130 / 80 \mathrm{mmHg}$ que no está confirmada por las medidas en el monitoreo ambulatorio de la presión arterial. El monitoreo ambulatorio ha sido ampliamente utilizado en el diagnóstico de hipertensión arterial en adultos, pero hay mucha menos experiencia con este método en niños. Se basa en el principio de que la medición repetida de la presión arterial durante las $24 \mathrm{~h}$ promueve una mejor aproximación al verdadero nivel de presión arterial que cuando se usa una sola medida. Es ventajoso en la evaluación del tratamiento antihipertensivo.

Después del diagnóstico, la hipertensión arterial suele clasificarse en dos formas: la hipertensión primaria o esencial, cuya causa se desconoce, y la hipertensión secundaria, en la que es posible identificar una patología responsable, para ser exactos, es posible encontrar una enfermedad / condición asociada que sea la causa real de la misma. Algunos ejemplos de tales enfermedades / condiciones: apnea del sueño, enfermedad renal crónica, síndrome de Cushing (exceso glucocorticoide), feocromocitoma (tumores que se originan en células de la glándula suprarrenal), hiperaldosteronismo primario, coartación aortica (estrechamiento de la aorta), enfermedad de tiroides y paratiroides, hipertensión renovascular, uso de anticonceptivos orales y el embarazo (Moura, Silva, \& Silva, 2015).

Hay indicios de que el tipo primario corresponde a alrededor del 95\% de los casos de hipertensión arterial, mientras que la secundaria representa el otro 5\% siendo las enfermedades renales y endocrinas las principales causas (Massierer, Oliveira, \& Steinhorst, 2012).

Cabe señalar, que no hay pruebas de diagnóstico ideales. Establecer un diagnóstico es un proceso susceptible de sistemática y errores aleatorios, lo que resulta en la probabilidad de certeza, y no la certeza en sí misma. El descubrimiento de la hipertension arterial en la mayoría de las personas, ocurre de manera casual sin la presencia de una queja específica, lo que demuestra a través 
de exámenes periódicos de salud, exámenes pre quirúrgicos, seguimiento durante el embarazo, en campañas de salud pública y a través de la medición de la presión arterial en farmacias y centros de salud. El diagnóstico es complejo porque está influenciado por la variabilidad de la presión arterial, equipos y técnicas usadas, posición corporal, hora del día, entorno, medida ubicación y por el responsable de la medición. Se observa que en niños pequeños (menores de seis años de edad) la hipertensión arterial tiene una mayor prominencia de ser secundaria, mientras que al final de la primera década y principios de la segundo, la hipertension comienza a ser de etiología predominante. Sin embargo, otros estudios afirman que la etiología de la hipertension arterial en niños parece seguir parámetros similares a los adultos, como la mayor frecuencia de casos de forma primaria, así como la falta de signos y síntomas que explican la presencia de la enfermedad. En esas circunstancias, el real problema de salud pública establecido por la hipertensión arterial se centra en la forma primaria de la enfermedad, ya que en la mayoría de los casos la etiología es difícil de detectar.

\section{Prevalencia}

La prevalencia de la hipertensión arterial ha ido en aumento en países desarrollados, por ser una enfermedad asintomática en sus primeras etapas y también la falta de información en la población, que afecta no solo a los ancianos sino también a individuos de grupos de edad cada vez más tempranas (Moura, Silva, \& Silva, 2015). En Portugal, la hipertensión arterial tiene una alta prevalencia superior al $40 \%$ en la población adulta y aunque en este grupo de edad predomina la hipertensión arterial, en niños y adolescentes no son despreciables.

Varios estudios también indicaron que la hipertensión arterial es una enfermedad con alta prevalencia en la población anciana, convirtiendose en un factor determinante en las altas tasas de 
morbilidad y mortalidad de estos individuos. Afecta a casi el $60 \%$ de los ancianos y se asocia a menudo con otras enfermedades tales como arteriosclerosis, diabetes mellitus y síndrome metabólico, confiriendo a este grupo un alto riesgo cardiovascular. Según la Dirección de Salud portuguesa, a través de un Estudio transversal realizado en 2013 (Macedo \& Ferreira, 2013) con individuos en edad igual o superior a 18 años, ha habido una mayor prevalencia de hipertensión arterial en el género femenino $(29,5 \%)$ y en la edad igual o superior a 65 años. Respecto a un estudio de base poblacional desarrollado en Brasil (Costa, Barcellos, \& Sclowitz, 2007) (individuos de 20 a 69 años), los datos mostraron una prevalencia de hipertensión arterial del 23,6\%, y que los individuos con menos de cuatro años de escolarización fueron casi el doble más probables de presentar hipertensión arterial; el género femenino exhibió un 17.0\% más de probabilidades de tener hipertensión arterial que el género masculino; en cuanto a la distribución de la hipertensión arterial con la edad, una tendencia significativa y positiva se encontró, es decir, la enfermedad aumenta con la edad; en relación al color de la piel, se verificó que las personas clasificadas como no-caucásicas presentó $25.0 \%$ más de hipertensión que los individuos de la categoría base (caucásicos). Otro estudio brasileño declaró que esta prevalencia en los pueblos originarios de África se debe al bajo nivel socioeconómico, a la ingesta de una dieta alta en sodio / baja en potasio y a la mala alimentación materna que conduce al bajo peso del niño al nacer, y esto en a su vez se asocia con el riesgo de desarrollar hipertensión arterial en la edad adulta.

\section{Factores de riesgo}

Los factores de riesgo cardiovascular se pueden clasificar en dos tipos: Modificable y no modificable. Los primeros son los que desde la perspectiva de la prevención podemos intervenir y corregir, e incluyen fumar, dislipidemia (colesterol alto), diabetes Mellitus, obesidad, inactividad

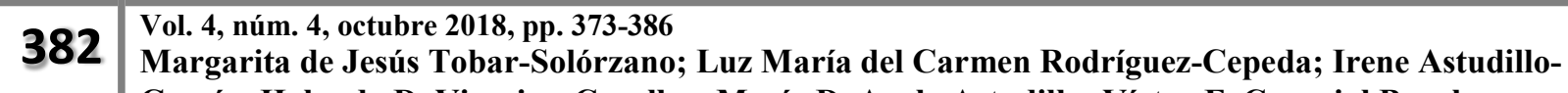
García; Holanda D. Vizcaino-Cevallos; María D. Ayala Astudillo; Víctor F. Carvajal Barahona
} 
física, exceso de alcohol, alto consumo de sodio, entre otros. Un estilo de vida saludable tiene una influencia positiva en todos estos factores de riesgo. Ya los factores de riesgo no modificables no están sujetos a intervención, e involucran la historia personal y familiar de la enfermedad cardiovascular.

Algunos autores afirman que la presión arterial de un individuo está determinada por la interacción entre el medio ambiente y factores genéticos (herencia), que generalmente se refieren a los factores modificables y no modificables, respectivamente.

En cuanto a los factores hereditarios, los estudios desarrollados en la era pre-molecular (Ferreira \& Aydos, 2010) han demostrado que estos factores contribuyen al menos el 20\% a 50\% de la variación de la presión arterial en humanos.

En cuanto a los factores ambientales, son igualmente importantes en determinar la presión arterial a lo largo de la vida. Algunos estudios declaran vigorosamente que uno o más de estos factores pueden cambiar permanentemente los factores fisiológicos, predisponiendo al individuo a un mayor riesgo de enfermedad cardiovascular más tarde. Para una mejor comprensión, los factores de riesgomas importantes y discutidos se dividieron en dietética y otros.

\section{Factores dietéticos}

Los factores dietéticos incluyen: sodio, potasio y obesidad, que son los más frecuentemente citados como factores de riesgo importantes para la hipertension arterial en la mayoría de los estudios. En cuanto al sodio, existe una relación bien documentada entre ingesta de sodio e hipertensión arterial en humanos (Ferreira \& Aydos, 2010). Algunos estudios también han demostrado que, asociado con el factor genético, la ingesta de sodio conduce a un rápido aumento

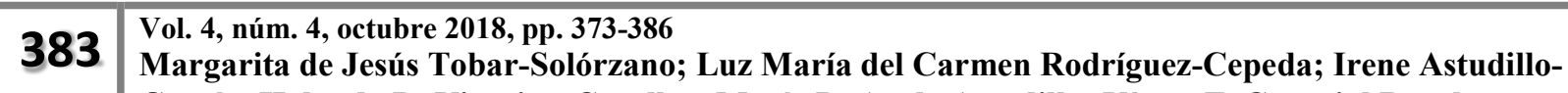
García; Holanda D. Vizcaino-Cevallos; María D. Ayala Astudillo; Víctor F. Carvajal Barahona
} 
de la presión arterial. Se ha encontrado una correlación positiva entre la ingesta de sodio y la presión arterial, después de ajustar por edad, género, cuerpo. indice de masa corporal (IMC), potasio y consumo de alcohol.

Sin embargo, un alto consumo de sodio no es suficiente para el desarrollo de la hipertensión arterial, ya que no todas las personas con alto contenido de sodio en la dieta la desarrollan. Este fenómeno se llama sensibilidad de sodio, esta sensibilidad es más evidente en pacientes con hipertensión severa, pacientes obesos, personas con antecedentes familiares de hipertension, en ancianos.

Los resultados de diferentes autores sugirieron que los niveles bajos de ingesta de potasio en la dieta puede ser una función importante en la génesis de hipertensión arterial. En adultos, la analogía de la obesidad y la hipertensión arterial puede ser detectada temprano en la infancia y tiene más relevancia clínica debido a la relación con enfermedades silenciosas como la dislipidemia y diabetes mellitus tipo II. Al comparar la hipertensión en personas de peso adecuado y sobrepeso, los individuos con obesidad tenían 3.5 veces más probabilidades de hipertensión arterial.

\section{Otros factores}

Factores como el estilo de vida sedentario, el estrés, las enfermedades, el tabaquismo y la ingesta de alcohol también influyen en el desarrollo de hipertensión arterial, que son las más preocupantes entre los factores modificables. Se ha encontrado que los fumadores tienen una mayor tasa de prevalencia que los no fumadores, y las personas consumidoras de alcohol son mas propensas a exhibir $\mathrm{AH}$ que aquellas que no consumen; Las personas que reportaron tener diabetes mellitus han mostrado mayor prevalencia de hipertensión arterial. Lo mismo se observa también en

\footnotetext{
384 Vol. 4, núm. 4, octubre 2018, pp. 373-386
Margarita de Jesús Tobar-Solórzano; Luz María del Carmen Rodríguez-Cepeda; Irene AstudilloGarcía; Holanda D. Vizcaino-Cevallos; María D. Ayala Astudillo; Víctor F. Carvajal Barahona
} 
varios estudios, tales como (Akter, Fauzi, Nordin, Satwi, \& Mohamed, 2010), en el que la hipertensión arterial también fue más común en individuos profesionalmente inactivos, diabéticos y entre aquellos con antecedentes familiares con enfermedades cardiovasculares; y en un estudio de ancianas, la cardiopatía y la diabetes fueron asociadas significativamente a estos factores ya que sus ocurrencias son más probables de exhibir alta presión sanguínea.

El consumo de alcohol fuera de las comidas aumenta el riesgo de hipertensión arterial, independientemente de la cantidad de alcohol consumido. Con respecto al estrés, esto contribuye a un gran número de enfermedades, tanto de naturaleza psíquica como orgánica, tales como la hipertensión.

\section{Conclusión.}

En conclusión, en esta revisión sistemática de la literatura, la hipertensión arterial afecta indiscriminadamente a todo el espectro de la población, aunque algunos grupos tienen características especiales, tanto en cuanto a su prevalencia en cuanto al pronóstico, como a la respuesta a diversas modalidades terapéuticas. Las situaciones especiales incluyen: género femenino, adultos y personas mayores, afrodescendientes, pueblos con educación inferior, enfermedades genéticas y factores ambientales (obesidad, alto consumo de sodio, estilo de vida sedentario, estrés, consumo de alcohol y hábitos de fumar). De esta manera, los objetivos propuestos en esta revisión han sido logrados exitosamente.

A pesar de la gran variación en la prevalencia de la hipertensión arterial, se observa que tales estudios son importantes para ayudar en políticas y acciones de salud pública, dando evidencia para

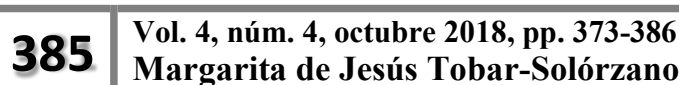


combatir la creciente prevalencia de la hipertensión en grupos de edades más jóvenes, contribuyendo así a la prevención de los factores de riesgos cardiovasculares en la edad adulta.

Sin embargo, las comparaciones entre diferentes estudios deben realizarse con precaución, sabiendo que la hipertensión arterial se limitaba a adultos mayores durante la mayor parte del siglo $\mathrm{XX}$, se ha encontrado que es necesario cambiar el enfoque actual, porque ahora esta enfermedad afecta a niños y adolescentes obesos en edades tempranas, lo que puede conducir a complicaciones cardiovasculares graves a una edad temprana, comprometiendo la calidad y la esperanza de vida de estas personas. Por lo tanto, es apropiado verificar la presencia de dichos factores de riesgo en una población más joven para prevenir enfermedades más graves que pueden ser instaladas tempranamente, reflejando muerte prematura en la población.

\section{Bibliografía.}

Akter, S., Fauzi, A., Nordin, M., Satwi, S., \& Mohamed, A. (2010). Prevalence of cardiovascular risk factors. PLoS One, 11(8), e0160180.

Costa, J., Barcellos, F., \& Sclowitz, M. (2007). Prevalência de Hipertensão Arterial em Adultos e Fatores Associados. Arquivos Brasileiros de Cardiologia, 88(1), 54-59.

Ferreira, J., \& Aydos, R. (2010). Prevalencia de hipertensión arterial. Rev. Latino-Am. Enfermagem, 22(4), 547-53.

Macedo, M., \& Ferreira, R. (2013). La hipertensión en Portugal: análisis epidemiológico en la atención primaria de salud.

Mariana, B., \& Pereira, P. (2014). Prevalencia de hipertensión arterial en adultos.

Massierer, D., Oliveira, A., \& Steinhorst, A. (2012). Prevalencia de Hipertensión Resistente en Adultos. Acta Med Colomb, 39(1), 57-60.

Moura, I., Silva, G., \& Silva, A. (2015). Prevalencia de hipertensión arterial y sus factores de riesgo en adolescentes.

Murray, C., \& Lopez, A. (1996). Global burden of disease and injury. Washington : OMS.

OMS. (2007). Prevencion de enfermedades cardiovasculares. Organizacion Mundial de la salud.

Salgado, C. (2007). Hipertension arterial en la infancia. Rev Cubana Pediatr, 88(3). 